\title{
Autonomous Planning of Multigravity-Assist Trajectories with Deep Space Maneuvers Using a Differential Evolution Approach
}

\author{
Ossama Abdelkhalik \\ Mechanical Engineering-Engineering Mechanics Department, Michigan Technological University, 815 R.L. Smith Building, \\ 1400 Townsend Dr., Houghton, Mine 49931-1295, USA
}

Correspondence should be addressed to Ossama Abdelkhalik; ooabdelk@mtu.edu

Received 2 February 2013; Revised 21 June 2013; Accepted 7 July 2013

Academic Editor: Paolo Tortora

Copyright (c) 2013 Ossama Abdelkhalik. This is an open access article distributed under the Creative Commons Attribution License, which permits unrestricted use, distribution, and reproduction in any medium, provided the original work is properly cited.

The biologically inspired concept of hidden genes has been recently introduced in genetic algorithms to solve optimization problems where the number of design variables is variable. In multigravity-assist trajectories, the hidden genes genetic algorithms demonstrated success in searching for the optimal number of swing-bys and the optimal number of deep space maneuvers. Previous investigations in the literature for multigravity-assist trajectory planning problems show that the standard differential evolution is more effective than the standard genetic algorithms. This paper extends the concept of hidden genes to differential evolution. The hidden genes differential evolution is implemented in optimizing multigravity-assist space trajectories. Case studies are conducted, and comparisons to the hidden genes genetic algorithms are presented in this paper.

\section{Introduction}

A fundamental step in the planning of interplanetary space missions is the design of the spacecraft trajectory. The multigravity-assist trajectory with deep space maneuvers (MGADSMs) is a trajectory that benefits from the gravitational fields of other planets to attain a free momentum change, by performing swing-bys around the planets. MGADSM trajectories can also use impulsive thrust to apply deep space maneuvers (DSMs) as needed. A criterion that is usually used in designing the MGADSM trajectory is to find the MGADSM trajectory that has minimum fuel expenditure. Designing the MGADSM trajectory is then formulated as an optimization problem. The design parameters that need to be optimized are the number of swing-bys, the planets to swing by, the times of swing-bys, the number of DSMs, the components and directions of these DSMs, the times at which these DSMs are applied, and the exact launch and arrival dates. This optimization problem, in its general form, is a variable-length optimization problem where the number of design variables is a variable. For instance, one solution may have 2 DSMs, and another solution for the same problem may have 3 DSMs. The number of design variables is different in both cases. The different number of swing-bys also causes the number of design variables to vary among different solutions.

Several optimization approaches have been proposed to solve the MGADSM trajectory design problem. Reference [1] developed a deterministic search space pruning algorithm to search for the optimal solution for a simplified version of the problem. When the gravity-assist sequence (i.e., the number of swing-bys and the planets to swing by) is known a priori, and assuming no DSMs, a pruning technique was developed to locate efficiently all the interesting parts in the search space of the multigravity-assist (MGA) variables [1]. This is called the MGA problem. Reference [2] implemented a stochastic initialization procedure, combined with a local optimization tool, to provide a set of locally optimal solutions to the same problem. The primer vector theory was extended to find the optimal number of DSMs as well as their magnitudes and directions [2]. In this method, the user specifies the sequence of swing-bys a priori. Reference [3] introduced relaxing boundary conditions to apply the interaction prediction principle to decompose the problem into subproblems. Parallel subproblems could then be solved. The algorithm was able to efficiently calculate the optimum number of DSM impulses and their locations. 
Evolutionary algorithms have been extensively investigated to search for the optimal solutions for the MGADSM problem. Reference [4] implemented a systematic branching strategy along with an evolutionary algorithm to balance the search between the local convergence and the global search. At each new run, the search space was reduced by performing a deterministic step. While the focus in [4] is on the balance between the local and global search when the number of variables is fixed [4] presented examples where the number of swing-bys is variable and the different MGA scenarios were explored. For the complex trajectories, Cassini and Rosseta [4] used wide ranges of design variables based on a priori knowledge of the solution space (assumed a fixed planet sequence). Reference [5] implemented a method inspired by the ant colony optimization to provide the optimal planetary sequence and a good estimation of the set of associated optimal trajectories. In [5], the trajectory model consists of a sequence of celestial bodies connected by two dimensional transfer arcs containing one DSM. Reference [6] implemented the differential evolution (DE) optimization method for the MGADSM problem. Reference [6] focused on the tuning of the algorithm parameters to improve the DE's performance and solved complex interplanetary missions, such as Cassini and Galileo.

Among the evolutionary algorithms are the genetic algorithms (GAs). GAs have been used widely in the literature to solve several orbital mechanics problems [7-11]. In order to implement the standard GAs in the MGADSM problem, the number of design variables needs to be fixed. This means that the number of swing-bys and the number of DSMs need to be known a priori. Recently, the concept of hidden genes in genetic algorithms (HGGA) was introduced to search for optimal solutions when the number of design variables is not fixed [12]. The HGGA is a biologically inspired concept where some of the genes are hidden to represent variables that are not part of a solution but are part of other solutions. The HGGA was implemented to the full MGADSM problem where no information about the number of swing-bys or the number of DSMs is known a priori $[13,14]$. DEs however have proven to be more efficient than GAs in searching for the optimal solutions of MGA problems, when the number of swing-bys and the number of DSMs are known a priori $[6,15]$.

In this paper, the concept of hidden genes is implemented in DE and applied to the MGADSM problem. Section 2 presents a brief description for the problem formulation. Section 3 describes how the DE works in the presence of hidden genes. The optimization approach for the MGADSM problem is detailed in Section 4. Numerical case studies are presented in Section 5.

\section{Problem Formulation}

A two-body dynamic model is assumed to govern the spacecraft motion at any time. The trajectory design problem is formulated as an optimization problem as follows: find the minimum cost selections for the number of swing-bys, the planets to swing by, the times of swing-bys, the number of DSMs, the components and directions of these DSMs, the

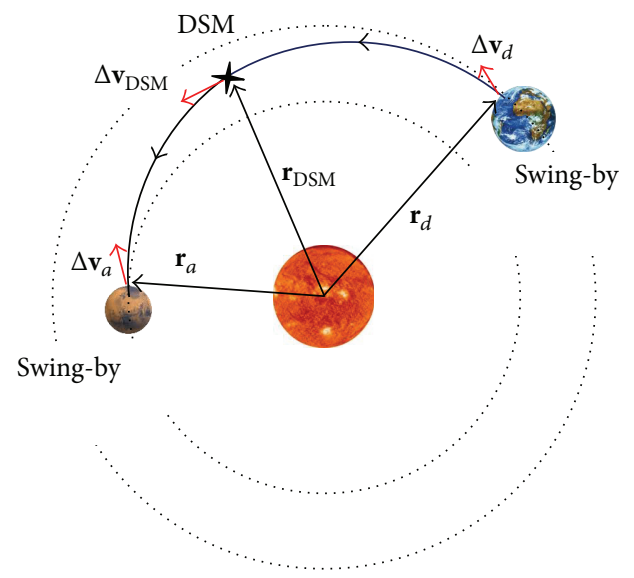

FIGURE 1: Three-impulse transfer orbit.

times at which these DSMs are applied, and the exact launch and arrival dates, given ranges for departure and arrival dates from the departure planet to a target planet. The cost is the total $\Delta v_{T}$ required for the mission. The spacecraft will benefit from as many as needed swing-bys of other planets. The segment between any two planets is called a leg. A leg can have any number of DSMs. Orbital mechanics details about how to compute the cost function from the design variables can be found in several references, for example, [13].

An essential step in any evolutionary optimization algorithm is to evaluate the cost function at a given design point. Some of the problem variables are independent, and some are dependent. At each design point, the optimization algorithm selects values for all the independent design variables while the dependent variables and the cost are to be calculated. These calculations are available in several references [13, 16-18] and are briefed in this section for completeness of presentation.

Assume $m$ gravity-assist maneuvers in a given mission (hence $m+1$ legs in the trajectory). Each leg contains $n_{l}$ deep space maneuvers. If a leg has one DSM or more, then the swing-by maneuver at the beginning of that leg is assumed to be a nonpowered swing-by maneuver [18]. If a leg has no DSM, then the swing-by at the beginning is assumed to be a powered swing-by maneuver. Figure 1 is a typical illustration for the geometry of a three-impulse leg in a trajectory. The time of flight for each leg, except the last leg, is an independent design variable [18].

In the nonpowered swing-by, the independent design variables associated with the swing-by are the normalized pericenter altitude $\bar{h}$ and a rotation angle $\eta$ [13]. The outgoing spacecraft velocity vector is then calculated using the swingby model. This velocity vector is the spacecraft heliocentric velocity vector of the initial point on the consequent transfer trajectory. The pericenter altitudes $h$ are normalized with respect to the mean radius of the associated swing-by planet, $R_{p}$. In the powered swing-by model, however, the dependent variables associated with the leg before the powered swing-by are first calculated. Next, the dependent variables associated with the leg after the powered swing-by are computed [13]. 
Finally, the powered swing-by variables, including the swingby impulse $\Delta \mathbf{v}_{p s}$, are computed such that the calculations of the two legs are compatible.

The times of swinging by planets are known (design variables provided by the optimizer); hence the positions of the spacecraft during the swing-by maneuvers are known. A Lambert solver is used to calculate the spacecraft velocity vectors on the initial and final points of the zero-DSM consequent leg. The velocity vector at the initial point of this leg is the required heliocentric outgoing velocity vector $\left(\mathbf{v}_{s / c}^{+}\right)_{\text {req }}$ of the powered swing-by at the associated swing-by planet [18]. To achieve this velocity, a swing-by impulse $\Delta \mathbf{v}_{p s}$ is added. To reduce the cost of the swing-by impulse, it is assumed that the powered swing-by impulse, the incoming relative velocity vector $\mathbf{v}_{\infty}^{-}$, and the required outgoing relative velocity vector $\left(\mathbf{v}_{\infty}^{+}\right)_{\text {req }}$ are in the same plane so that $\Delta \mathbf{v}_{p s}$ does not have an out-of-plane component

$$
\left(\mathbf{v}_{\infty}^{+}\right)_{\text {req }}=\left(\mathbf{v}_{s / c}^{+}\right)_{\text {req }}-\mathbf{v}_{p} .
$$

The $\mathbf{v}_{\infty}^{+}$is calculated as

$$
\mathbf{v}_{\infty}^{+}=\mathbf{v}_{\infty} \frac{\left(\mathbf{v}_{\infty}^{+}\right)_{\text {req }}}{\left\|\left(\mathbf{v}_{\infty}^{+}\right)_{\text {req }}\right\|} .
$$

\section{Differential Evolution with Hidden Genes Chromosomes}

DE is a population based function optimization algorithm. It encodes all the design variables into a chromosome. The initial population of chromosomes (solutions) is randomly generated. After initialization, the DE enters a loop of evolutionary operations; these operations are the mutations, crossover, and selection [19]. A population consists of NP members, and each member is of dimension $D$. So, at each generation, $g$, the population is $\mathbf{x}_{i, g}=\left[x_{1, i, g}, x_{2, i, g}, \ldots, x_{D, i, g}\right]^{T}, i=1,2, \ldots, \mathrm{NP}$. The mutation operation uses the current population to create trial vectors $\mathbf{v}_{i, g}$. There are different strategies for mutation in the literature one of them is defined as

$$
\mathbf{v}_{i, g}=\mathbf{x}_{r 0, g}+F_{i}\left(\mathbf{x}_{r 1, g}-\mathbf{x}_{r 2, g}\right),
$$

where $r 0, r 1$, and $r 2$ are three different integers uniformly selected from the set $1,2, \ldots, \mathrm{NP}$, and $F_{i}$ is the mutation factor [20]. After mutation, the crossover operation forms the final trial vector $\mathbf{u}_{i, g}=\left[u_{1, i, g}, u_{2, i, g}, \ldots, u_{D, i, g}\right]^{T}$, where $[20]$

$$
u_{j, i, g}= \begin{cases}v_{j, i, g}, & \text { if } \operatorname{rand}_{j}(0,1) \leq \mathrm{CR} \text { or } j=j_{\text {rand }}, \\ x_{j, i, g}, & \text { otherwise }\end{cases}
$$

where $\operatorname{rand}_{j}(0,1)$ returns a uniform random number on the interval $[0,1], j_{\text {rand }}$ is an integer randomly chosen from 1 to $D$, and the crossover probability is $\mathrm{CR} \in[0,1]$. The selection operation selects the more fit one from the parent and the trial vectors, $\mathbf{x}_{i, g}$ and $\mathbf{u}_{i, g}$, respectively.

The hidden genes implementation in differential evolution is not unlike their implementation in genetic algorithms which is detailed in [13]. The hidden genes concept mimics

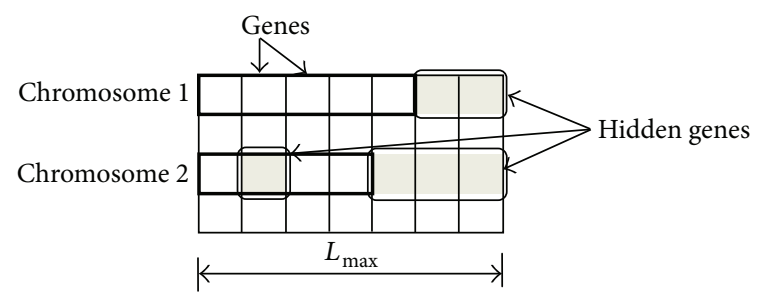

FIGURE 2: The addition of hidden genes makes all chromosomes become of equal length.

biology in that not all proteins are made in every cell, and hence not every gene is read in every cell [21]. For example, an eye cell does not need any breathing genes on. And so they are shut off in the eye. Seeing genes are also shut off in the lungs. Another layer of coding tells what genes a cell should read and what genes should be hidden from the cell [21].

Figure 2 shows two chromosomes of different lengths in a standard DE implementation. The mutation operation described in (3) cannot be carried out if standard DE is implemented because the lengths of the vectors $\mathbf{x}_{r 0, g}, \mathbf{x}_{r 1, g}$, and $\mathbf{x}_{r 2, g}$ are, in general, different. When hidden genes are added, the lengths of the chromosomes become the same $\left(L_{\max }\right)$. The hidden genes represent variables that do not exist in a specific solution but exist in others, as shown in Figure 2. For example, consider the MGADSM problem where the maximum possible number of swing-bys allowed in the mission is 5 . A specific solution for the mission might have only 2 swing-bys. The chromosome representing this solution will have hidden genes representing the variables associated with 3 swing-bys. These hidden genes do not affect the fitness of the solution. However, they take part in the crossover and mutation operations, and hence they affect future generations.

The hidden genes differential evolution (HGDE) method is tested for several interplanetary missions ranging from simple to complex missions. The optimization algorithm finds the mission scenario (the number of swing-bys and the planets to swing by) as well as the rest of the independent design variables: the times of swing-by, the number of DSMs, the times of DSMs, the magnitudes/directions of DSMs, and the departure/arrival dates. The size of the design space is controlled by the bounds of the independent design variables.

\section{MGADSM Trajectory Optimization Using HGDE}

In this paper, the objective is to minimize the total cost, $\Delta v_{T}$, of the MGADSM trajectory, where

$$
\Delta v_{T}=\left\|\Delta \mathbf{v}_{d}\right\|+\sum_{1}^{m}\left\|\Delta \mathbf{v}_{p s}\right\|+\sum_{1}^{m+1} \sum_{1}^{n_{l}}\left\|\Delta \mathbf{v}_{\mathrm{DSM}}\right\|+\left\|\Delta \mathbf{v}_{a}\right\|
$$

where $m$ is the number of gravity-assist maneuvers, $n_{l}$ is the number of deep space maneuvers in each leg of the $m+1$ mission's legs, $\Delta \mathbf{v}_{d}$ and $\Delta \mathbf{v}_{a}$ are the departure and arrival impulses, respectively, $\Delta \mathbf{v}_{p s}$ is the postswing-by impulse of 
TABLE 1: The discrete and continuous independent design variables of the MGADSM problem.

\begin{tabular}{lc}
\hline $\begin{array}{l}\text { Continuous design variables } \\
(\mathrm{CDV})\end{array}$ & $\begin{array}{r}\text { Discrete design variables } \\
(\mathrm{DDV})\end{array}$ \\
\hline $\begin{array}{l}\text { Departure date, } t_{d} \\
\text { Arrival date, } t_{a}\end{array}$ & $\begin{array}{r}\text { No. of swing-by maneuvers, } m \\
\text { Swing-by planets, } P_{1}, \ldots, P_{i}\end{array}$ \\
Time of flight, $T_{1}, \ldots, T_{i}$ & $\begin{array}{r}\text { The count of DSMs in each leg, } \\
n_{1}, \ldots, n_{i+1}\end{array}$ \\
$\begin{array}{l}\text { Normalized pericenter altitudes, } \\
\bar{h}_{1}, \ldots, \bar{h}_{i}\end{array}$ & Flight direction, $f$ \\
Rotation angles, $\eta_{1}, \ldots, \eta_{i}$ & \\
Epochs of DSMs, $\varepsilon_{1}, \ldots, \varepsilon_{j}$ & \\
DSMs, $\Delta \mathbf{v}_{1}, \ldots, \Delta \mathbf{v}_{k}$ & \\
\hline
\end{tabular}

the powered gravity assist only, and $\Delta \mathbf{v}_{\text {DSM }}$ is the applied deep space maneuver impulse.

The HGDE is implemented for optimization to search for the optimal solution. Each chromosome represents the independent design variables. In this optimization problem, some of the design variables are continuous (CDVs) and some are discrete (DDVs), as seen in Table 1. In Table 1, $i$ is the maximum possible number of swing-bys, $j$ is the maximum possible number of total DSMs, and $k$ is the maximum possible number of powered swing-bys in the entire trajectory. The epoch of a DSM, $\varepsilon$, specifies the time at which the impulsive maneuver is applied, as a fraction of the associated leg transfer time. The flight direction $f$ is a binary variable that specifies the direction of flight around the Sun (prograde or retrograde).

In complex missions, the computational cost of the problem may be reduced by splitting the problem into two phases [13]. A trajectory design optimization can be started by assuming no DSMs in the trajectory (zero-DSM trajectory). This reduces the number of independent design variables by eliminating the following design variables: swing-bys periapsis altitudes, swing-bys rotation angles, epoch of DSMs, and thrust impulses. This reduction in the number of design variables allows exploring wider ranges for each of the remaining design variables. In the second step, DSMs are added to the obtained fit scenario (zero-DSM solutions). In the second step, we optimize the number of DSMs in each leg and their locations/magnitudes/directions, while maintaining the scenario fixed. The departure/arrival dates and times of flight are allowed to change as design variables, with narrow ranges around the values obtained from the zero-DSM solution. This technique of solving the problem by the two-phase approach has the advantage of reducing the computational time. Specifically, for the Cassini 2 mission trajectory, if the maximum number of swing-bys is four and only one impulse, as a maximum, is allowed in each leg, then the number of independent design variables is 33 (11 DDVs and 22 CDVs). This is a computationally expensive problem, especially with wide ranges for the design variables. The zeroDSM problem has only 12 design variables (6 DDVs and 6 CDVs). Then, the second step is performed with 27 design variables (5 DDVs and $22 \mathrm{CDVs}$ ). The ranges of the CDVs in the second step are reduced based on the information from the first step solution.

\section{Case Studies}

This section presents three case studies for interplanetary space missions trajectory optimization. Comparisons to other solutions in the literature are presented to validate the obtained results. A MATLAB code (available with [20]) is adopted in implementing the HGDE. DE has three main parameters that need to be tuned. These parameters are the population size (NP), mutation factor $(F)$, and the crossover probability (CR). For MGADSM problems, [6] presents a study on the tuning of the standard DE parameters and recommends a range for each parameter. These recommendations are adopted in this paper. Unless otherwise specified, the population size, the mutation factor, and the crossover probability are selected to be $30,0.8$, and 0.7 , respectively [6].

In order to test the HGDE algorithm, statistics are generated on its behavior over a number of runs and for a given computational effort (measured in terms of the number of function evaluations). Rigorous procedures have been developed for testing global optimization algorithms $[6,15,22]$. In this paper, the algorithm developed in [15] is implemented to test the capability of HGDE in finding the best cost solution. The algorithm presented in [15] measures the success rate of an algorithm in finding the best cost solution. Because the main advantage of the HGDE is its capability of finding the optimal sequence of planets, another measure is implemented to measure the success rate of the algorithm in finding a swing-by sequence [12]. The success rate $p_{s}$ is computed as $p_{s}=j_{s} / n$, where $n$ is the number of runs and $j_{s}$ is a counter that counts how many times the best sequence resulted as a solution at the end of an experiment. In some experiments, the algorithm produces a swing-by sequence that is not the best known solution. In this case, the success rate $p_{s}$ represents the success rate of the algorithm in finding the solution that it finds not the best known solution. For example, if the best know swingby sequence of a mission is (VEE) and the HGDE algorithm best solution has a sequence (EE), then the success rate $p_{s}$ represents the success rate in finding the solution (EE). Hence, $p_{s}$ is not sufficient in measuring the efficiency of the HGDE, and an additional metric is here introduced to quantify how an obtained swing-by sequence is far from the best known sequence. This swing-by sequence distance metric (SSDM) along with $p_{s}$ determines the efficiency of the HGDE in solving a given problem. The following section details how the SSDM is defined.

5.1. Swing-By Sequence Distance Metric. Suppose that the number of swing-by planets in a given solution $j$ is $S_{j}$, and the number of swing-by planets in the best known solution is $S_{B}$. Let

$$
a_{j}=\left(S_{j}-S_{B}\right)^{2}
$$

Hence, the term $a_{j}$ quantifies the error in the number of swing-by planets between an obtained solution $j$ and the best 
TABLE 2: Bounds of Earth-Mars mission's design variables using HGGA.

\begin{tabular}{lcc}
\hline Design variables & Lower bound & Upper bound \\
\hline $\begin{array}{l}\text { No. of swing-by maneuvers } \\
\text { Swing-by planets }\end{array}$ & 0 & 2 \\
$\begin{array}{l}\text { No. of DSMs per leg } \\
\text { Flight direction, } f\end{array}$ & 0 & 3 (Earth) \\
$\begin{array}{l}\text { Departure date, } t_{d} \\
\text { Arrival date, } t_{a}\end{array}$ & Prograde & 2 \\
Time of flight (days)/leg, & Retrograde \\
$T_{1}, \ldots, T_{i}$ & 4004 & 01-December-2004 \\
$\begin{array}{l}\text { Swing-by normalized } \\
\text { pericenter altitude, }, \bar{h}_{1}, \ldots, \bar{h}_{i}\end{array}$ & 0.8 & 01-July-2005 \\
$\begin{array}{l}\text { Swing-by plane rotation } \\
\text { angle (rad), } \eta_{1}, \ldots, \eta_{i}\end{array}$ & 0 & 300 \\
$\begin{array}{l}\text { Epoch of DSM, } \varepsilon_{1}, \ldots, \varepsilon_{j} \\
\text { DSM }(\mathrm{km} / \mathrm{s}), \Delta v_{1}, \ldots, \Delta v_{k}\end{array}$ & 0.1 & 5 \\
\hline
\end{tabular}

known solution; if the solution $j$ has the same number of swing-bys as the best known solution, then $a_{j}=0$. Let $b_{i}$ be an index associated with the $i$ th swing-by planet. If the $i$ th swing-by planet is the same in both the best known solution and the obtained solution $j$, then $b_{i}=0$, otherwise $b_{i}=1$. For example, the two sequences (EVE) and (EVV) have $b_{1}=0, b_{2}=0$, and $b_{3}=1$. Hence the quantity $c_{j}=\sum b_{i}$ quantifies how far a planets' sequence $j$ is from the best known sequence (assuming that both sequences have the same number of swing-bys; that is, $a_{j}=0$.) If two sequences have different lengths, then the longer sequence will be reduced in length to match the shorter sequence in order to compute $a_{j}$. The reduction in length can only be carried out at the beginning or/and at the end of the longer sequence. For example, consider the $j$ th sequence $(\mathrm{EV})$ and the sequence (EEVV). There are three possible values for $c_{j}$ resulting from comparing the $j$ th sequence $(\mathrm{EV})$ to each of the following subsets (EE), (EV), and (VV). The corresponding values of $c_{j}$ are $c_{j 1}=1, c_{j 2}=0$, and $c_{j 3}=1$, respectively. The value of $c_{j}$ will be selected to be the minimum of $c_{j l}$ for all $l$. The value of $l$ can be easily computed, $l=k+1$, where $k=$ minimum $\left(S_{j}, S_{B}\right)$. Hence the SSDM of a solution $j$ from the best known sequence $\left(\operatorname{SSDM}_{j}\right)$ is defined as

$$
\begin{gathered}
\operatorname{SSDM}_{j}=a_{j}+c_{j}, \quad \text { where } \\
c_{j}=\min \left(\sum_{i=l}^{k} b_{i}\right), \quad \forall l .
\end{gathered}
$$

5.2. Earth-Mars Mission Using HGDE. The HGDE is used to search for the optimal trajectory for the Earth-Mars mission. The objective is to obtain a minimum cost trajectory. All the design variables are optimized in one optimization step (the full MGADSM problem). The ranges of the design variables are shown in Table 2.

The total number of design variables for this mission is 33. A population of 400 individuals is used, for 600 iterations
TABLE 3: Solution trajectory for the Earth-Mars mission.

\begin{tabular}{lc}
\hline Mission parameter & Value \\
\hline Departure date, $t_{d}$ & 02-Jun-2004 00:07:31 \\
Departure impulse $(\mathrm{km} / \mathrm{s})$ & 4.3517 \\
DSM date & 16-Aug-2004 14:23:36 \\
DSM impulse $(\mathrm{km} / \mathrm{s})$ & 0.32836 \\
Venus swing-by date & 18-Nov-2004 02:34:34 \\
Pericenter altitude $(\mathrm{km})$ & 7890.1 \\
Arrival date & 16-May-2005 18:13:39 \\
Arrival impulse $(\mathrm{km} / \mathrm{s})$ & 6.0366 \\
Mission duration $(\mathrm{days})$ & 348.75 \\
\hline Mission cost $(\mathrm{km} / \mathrm{s})$ & $\mathbf{1 0 . 7 1 7}$ \\
\hline
\end{tabular}

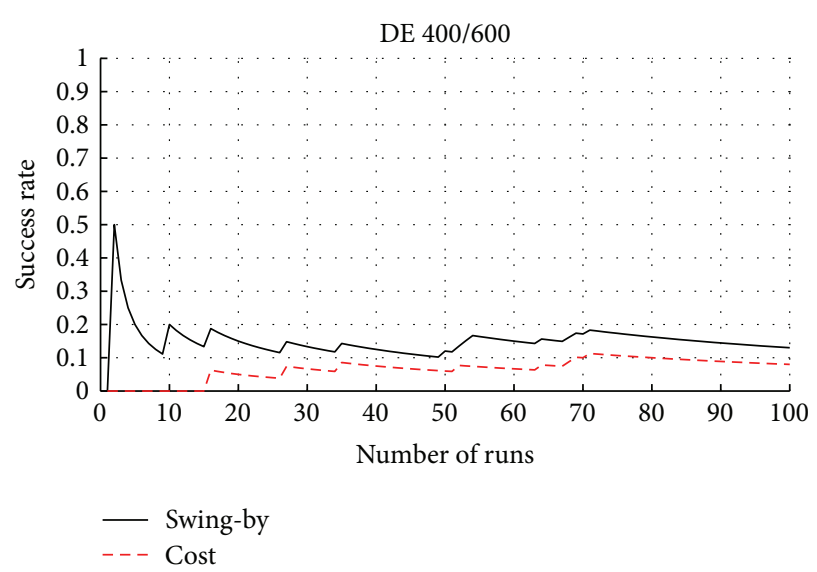

FIgURE 3: MGADSM success rates.

(DE 400/600). A local optimizer uses the fittest solution as an initial guess to find a local minimum. The resulting solution has a single swing-by maneuver at Venus, with a total cost of $10.716 \mathrm{~km} / \mathrm{s}$. It has a single DSM in the first leg as shown in Table 3. The obtained sequence is the same as the best known solution, and hence SSDM $=0$.

Statistics are generated on the behavior of the HGDE over 100 runs (for 240,000 function evaluations each). The success rate in finding the optimal sequence settles at $12 \%$, whereas the success rate in finding the minimum mission cost settles at 9\%, as shown in Figure 3. Section 6 compares these results with other methods in the literature. For the sake of comparison with other methods, the same problem is solved with a narrow launch date range (June 01, 2004-July $01,2004)$ to match that used in [13]. In this case, a DE 30/200 is used. The obtained solution is very close to that listed in Table 3 with a total mission cost of $10.73 \mathrm{~km} / \mathrm{s}$. The success rates in this case are $30 \%$ and $20 \%$ for the sequence and cost, respectively.

The same problem is solved again on two steps. The first step is the search for the zero-DSM trajectory, and the second step is the search for the optimal DSMs for the obtained zeroDSM trajectory in the first step. The lower and upper bounds for the first step are shown in Table 4. As can be seen in Table 4, a wide range for the swing-by planets is assumed, and no DSMs are assumed. This HGDE is run 25 times, 
TABLE 4: Bounds of Earth-Mars mission's design variables: first step.

\begin{tabular}{lcc}
\hline Design variables & Lower bound & Upper bound \\
\hline $\begin{array}{l}\text { No. of swing-by maneuvers } \\
\text { Swing-by planets }\end{array}$ & 0 & 2 \\
$\begin{array}{l}\text { Departure date, } t_{d} \\
\text { Arrival date, } t_{a}\end{array}$ & $\begin{array}{c}\text { 01-Jun-2004 } \\
\text { 01-Apr-2005 }\end{array}$ & $\begin{array}{c}\text { 01-July-2004 } \\
\text { 01-July-2005 }\end{array}$ \\
$\begin{array}{l}\text { Time of flight (days)/leg, } \\
T_{1}, \ldots, T_{i}\end{array}$ & 40 & 300 \\
$\begin{array}{l}\text { Swing-by normalized } \\
\text { pericenter altitude, }, \bar{h}_{1}, \ldots, \bar{h}_{i}\end{array}$ & 0.8 & 5 \\
$\begin{array}{l}\text { Swing-by plane rotation } \\
\text { angle (rad), } \eta_{1}, \ldots, \eta_{i}\end{array}$ & 0 & $2 \pi$ \\
DSM (km/s), $\Delta v_{1}, \ldots, \Delta v_{k}$ & -5 & 5 \\
\hline
\end{tabular}

TABLE 5: Both the zero-DSM and MGADSM solutions for the EarthMars mission.

\begin{tabular}{lcc}
\hline Mission parameter & Zero-DSM model & MGADSM model \\
\hline Departure date, $t_{d}$ & 05-Jun-2004 & 01-Jun-2004 \\
Departure impulse $(\mathrm{km} / \mathrm{s})$ & $15: 50: 23$ & $00: 00: 00$ \\
DSM date & 4.612 & 4.1985 \\
& - & $20-J u l-2004$ \\
DSM impulse $(\mathrm{km} / \mathrm{s})$ & - & $16: 56: 38$ \\
Venus swing-by date & $20-\mathrm{Nov}-2004$ & 0.43784 \\
Pericenter altitude $(\mathrm{km})$ & $11: 02: 26$ & $09: 36: 00$ \\
Arrival date & 8050 & 7962.5 \\
& $13-\mathrm{May}-2005$ & $16-\mathrm{May}-2005$ \\
Arrival impulse $(\mathrm{km} / \mathrm{s})$ & $16: 19: 11$ & $21: 07: 12$ \\
Mission duration $(\mathrm{days})$ & 6.1667 & 6.0906 \\
\hline Mission cost $(\mathrm{km} / \mathrm{s})$ & 342.02 & 349.88 \\
\hline
\end{tabular}

and in all the runs the HGDE found the optimal swing-by sequence which is (EVM). So, the success rate for finding the optimal swing-by in this case is $100 \%$. The rest of the solution parameters are shown in Table 5. The obtained sequence is the same as the best known solution, and hence SSDM $=0 . \mathrm{A}$ comparison between Tables 3 and 5 shows that the obtained solution in the first step is close to the solution obtained when the whole problem is solved in one step. The second step assumes the sequence is (EVM) and searches for the optimal DSM structure. The number of DSMs in each leg is assumed 0,1 , or 2 . The location of each DSM in a leg could be anywhere between 0.1 and 0.9 of the leg time of flight. The best solution found in the second step is shown in Table 5. The obtained solution is almost the same solution obtained in Table 3 . The success rate in finding the minimum cost is shown in Figure 4. The success rate settles at $90 \%$.

5.3. Jupiter Europa Orbiter Mission (JEO). The HGDE is used to investigate the JEO mission $[23,24]$. Table 6 shows the ranges of the design variables used for the zero-DSM model. In the MGADSM model, the zero-DSM solution swing-by sequence is used, and the ranges of the rest of the design
TABLE 6: Bounds on design variables for JEO mission-Zero-DSM model.

\begin{tabular}{lcc}
\hline Design variables & Lower Bound & Upper Bound \\
\hline No. of swing-by maneuvers & 1 & 4 \\
Swing-by planets & 2 (Venus) & 4 (Mars) \\
Flight direction, $f$ & Prograde & Retrograde \\
$\begin{array}{l}\text { Departure date, } t_{d} \\
\text { 01-Feb-2018 }\end{array}$ & 29-Mars-2018 \\
Arrival date, $t_{a}$ & 01-Jan-2026 & 30-Jun-2026 \\
Time of flight (days)/leg, & 200 & 1100 \\
$T_{1}, \ldots, T_{i}$ & & 10 \\
$\begin{array}{l}\text { Swing-by normalized } \\
\text { pericenter altitude, } \bar{h}_{1}, \ldots, \bar{h}_{i}\end{array}$ & 0.1 & \\
$\begin{array}{l}\text { Swing-by plane rotation } \\
\text { angle (rad), } \eta_{1}, \ldots, \eta_{i}\end{array}$ & 0 & $2 \pi$ \\
\hline
\end{tabular}

TABLE 7: Bounds on design variables for JEO mission: MGADSM model.

\begin{tabular}{lcc}
\hline Design variables & Lower bound & Upper bound \\
\hline No. of DSMs per leg & 0 & 1 \\
Flight direction, $f$ & Prograde & Retrograde \\
Departure date, $t_{d}$ & 20-Feb-2018 & 24-Feb-2018 \\
Arrival date, $t_{a}$ & 01-Feb-2026 & 28-Feb-2026 \\
Time of flight (days)/leg, & {$[350,700,800$,} & {$[450,800,900$,} \\
$T_{1}, \ldots, T_{i}$ & $850]$ & $950]$ \\
Epoch of DSM, $\varepsilon_{1}, \ldots, \varepsilon_{j}$ & 0.1 & 0.9 \\
DSM $(\mathrm{km} / \mathrm{s}), \Delta v_{1}, \ldots, \Delta v_{k}$ & -10 & 10 \\
\hline
\end{tabular}

variables are narrowed down around the zero-DSM solution, as shown in Table 7.

A population of 400 members and 600 iterations (DE $400 / 600$ ) is used, in both steps. The best zero-DSM solution found using the HGDE is a three-swing-by trajectory (EVEEJ), as shown in Table 8. Several zero-DSM solutions are found with very close costs and slight differences in departure and/or arrival dates and impulses. In these solutions, the total mission cost is about $9.2 \mathrm{~km} / \mathrm{s}$, and the mission duration is about 8 years. It is worth mentioning that other solutions, of lower cost (about $9.4 \mathrm{~km} / \mathrm{s}$ ) but with a mission duration of about 6.6 years. The cost function in this study, however, accounts only for the mission cost, and hence a shorter mission duration does not affect the fitness of a solution.

Statistics are generated on the behavior of the HGDE over a number of runs and for a given computational effort (measured in terms of the number of function evaluations). Two cases are considered: DE 500/60 and DE 400/600. The JEO mission problem is run 51 times (it has been observed that there is no significant variation in the success rate after 51 iterations for this case study so we chose to stop at 51). For the DE 500/60, the success rate settles at above $70 \%$ for finding the best swing-by sequence but settles at above $10 \%$ for finding the zero-DSM cost, as shown in Figure 5(a). The best solution found in this case has a planet sequence of EVEJ and a cost of $12.6 \mathrm{~km} / \mathrm{s}$. The SSDM for this solution is 1 . For the DE $400 / 600$, the success rate settles at $70 \%$ for finding 
TABLE 8: Optimal trajectory of Earth-Jupiter mission (EVEEJ) using HGDE.

\begin{tabular}{|c|c|c|}
\hline Mission parameter & Zero-DSM model & MGADSM model \\
\hline Planet sequence & EVEEJ & EVEEJ \\
\hline Departure date & 01-Mars-2018 11:53:57 & 26-Feb-2018 06:08:00 \\
\hline Departure impulse $(\mathrm{km} / \mathrm{s})$ & 3.4952 & 2.887 \\
\hline DSM date & - & 10-Dec-2018 08:40:45 \\
\hline DSM impulse $(\mathrm{km} / \mathrm{s})$ & - & 0.38609 \\
\hline Venus swing-by date & 10-Apr-2019 11:51:46 & 01-Apr-2019 05:27:15 \\
\hline Postswing-by impulse $(\mathrm{km} / \mathrm{s})$ & 0.0 & 0.0 \\
\hline Earth swing-by date & 20-Oct-2021 03:45:19 & 19-Oct-2021 18:56:15 \\
\hline Post-swing-by impulse $(\mathrm{km} / \mathrm{s})$ & 0.0 & 0.0 \\
\hline Earth swing-by date & 19-Jun-2023 19:03:28 & 18-Jun-2023 23:16:31 \\
\hline Post-swing-by impulse $(\mathrm{km} / \mathrm{s})$ & 0.0 & 0.0 \\
\hline Arrival date & 01-Mar-2026 16:05:27 & 16-Feb-2026 06:58:55 \\
\hline Arrival impulse (km/s) & 5.688 & 5.693 \\
\hline Time of flight (days) & 404.99-923.66-607.64-985.88 & $399-932.6-607.2-973.3$ \\
\hline Mission cost $(\mathrm{km} / \mathrm{s})$ & 9.19 & 8.97 \\
\hline
\end{tabular}

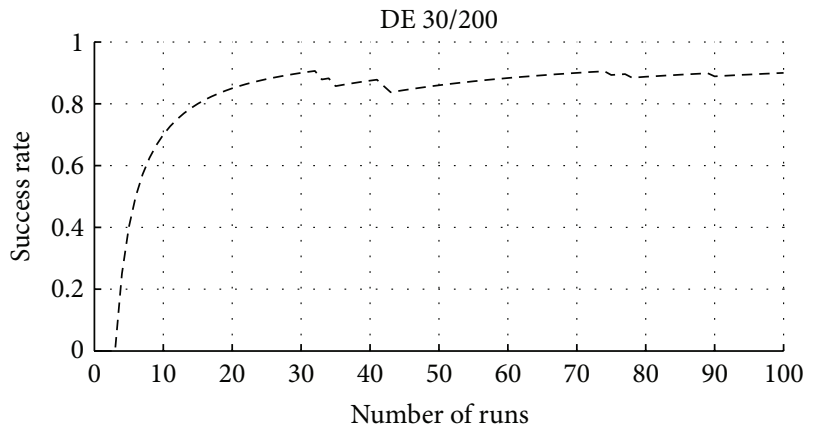

FIgURE 4: Zero-DSM case: Cost success rate.

the optimal swing-by but settles at $64 \%$ for finding the zeroDSM cost, as shown in Figure 5(b). The best solution found in this case has a planet sequence of EVEEJ. The SSDM for this solution is 0 .

In the second stage, the planet swing-by sequence is fixed at EVEEJ, and the optimal DSMs are calculated. The number of variables in the second stage for the JEO mission is 23 . The minimum cost found using HGDE is $8.97 \mathrm{~km} / \mathrm{s}$. Figure 6(a) shows the success rate in finding the minimum cost in the second stage. The success rate settles at 50\% after 100 runs. The number of function evaluations in this statistical study is 100,000 . Table 8 shows the details of the obtained solution. After departure, the spacecraft completes one revolution around the Sun before it swings by Venus. The trajectory has one DSM in the first leg. After the Venus swingby, the spacecraft completes one revolution around the Sun before it swings by the Earth. The third swing-by is around the Earth, after which the spacecraft flies toward Jupiter, as shown in Figure 6(b).

5.4. Earth-Saturn Mission (Cassini 2). The Cassini 2 mission trajectory is one of the most challenging multigravity-assist trajectories to be optimized. The target is to study the planet
TABLE 9: Bounds on design variables for Cassini 2 mission: zeroDSM model.

\begin{tabular}{lcc}
\hline Design variables & Lower bound & Upper bound \\
\hline $\begin{array}{l}\text { No. of swing-by maneuvers, } \\
m\end{array}$ & 1 & 4 \\
$\begin{array}{l}\text { Swing-by planets } \\
\text { identification numbers, } \\
P_{1}, \ldots, P_{i}\end{array}$ & 2 (Venus) & 5 (Jupiter) \\
$\begin{array}{l}\text { Flight direction, } f \\
\text { Departure date, } t_{d}\end{array}$ & Prograde & Retrograde \\
$\begin{array}{l}\text { Arrival date, } t_{a} \\
\text { Time of flight }(\text { days)/leg, }\end{array}$ & 01-Nov-1997 & 31-Nov-1997 \\
$T_{1}, \ldots, T_{i}$ & 01-Jan-2007 & 30-Jun-2007 \\
$\begin{array}{l}\text { Swing-by normalized } \\
\text { pericenter altitude, } \bar{h}_{1}, \ldots, \bar{h}_{i}\end{array}$ & 0.1 & 1000 \\
$\begin{array}{l}\text { Swing-by plane rotation } \\
\text { angle (rad), } \eta_{1}, \ldots, \eta_{i}\end{array}$ & 0 & 10 \\
$\begin{array}{l}\text { Epoch of DSM, } \varepsilon_{1}, \ldots, \varepsilon_{j} \\
\text { DSM (km/s), } \Delta v_{1}, \ldots, \Delta v_{k}\end{array}$ & 0.1 & $2 \pi$ \\
\hline
\end{tabular}

Saturn and its moons [25]. The HGDE tool is used to search for a minimum cost trajectory from Earth to Saturn. For the sake of making comparisons with the literature, a narrow range of departure date is allowed, around the known published date for the Cassini 2 mission. The ranges for the other design variables are wide enough to investigate all possible solutions. Table 9 presents the upper and lower bounds for the design variables. First, the problem is investigated assuming no DSMs. For this zero-DSM model, the population size is selected to be NP $=500$, and the number of iterations is 200 (DE 500/200).

The best solution found in 200 runs has two swingby maneuvers at Venus and Jupiter, (Earth-Venus-JupiterSaturn (EVJS)). This is different from the actual Cassini 2 mission scenario (Earth-Venus-Venus-Earth-Jupiter-Saturn 


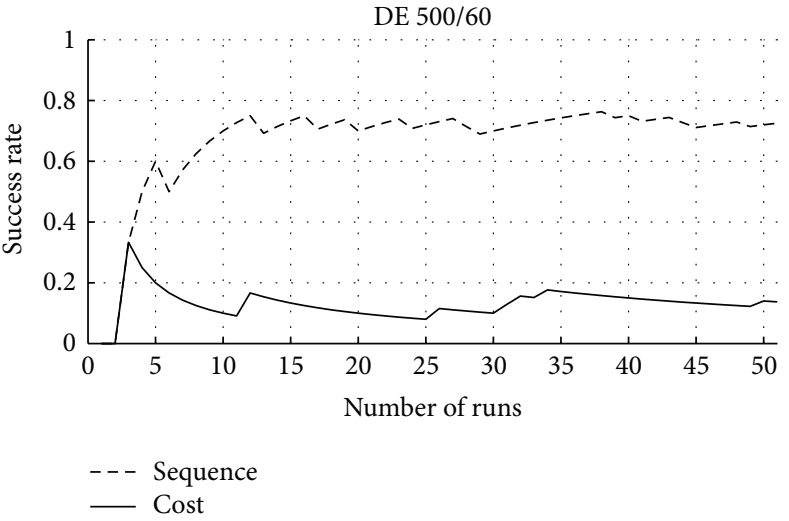

(a) $\mathrm{EVEJ} / \mathrm{cost}=12.6 \mathrm{~km} / \mathrm{s}$

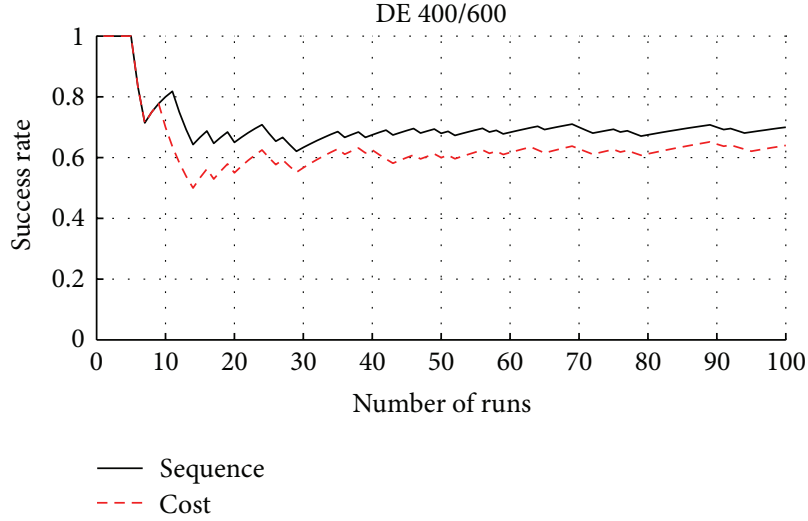

(b) EVEEJ $/$ cost $=9.19 \mathrm{~km} / \mathrm{s}$

FIGURE 5: The success rate of finding the minimum cost trajectory versus the number of runs for the JEO zero-DSM case.

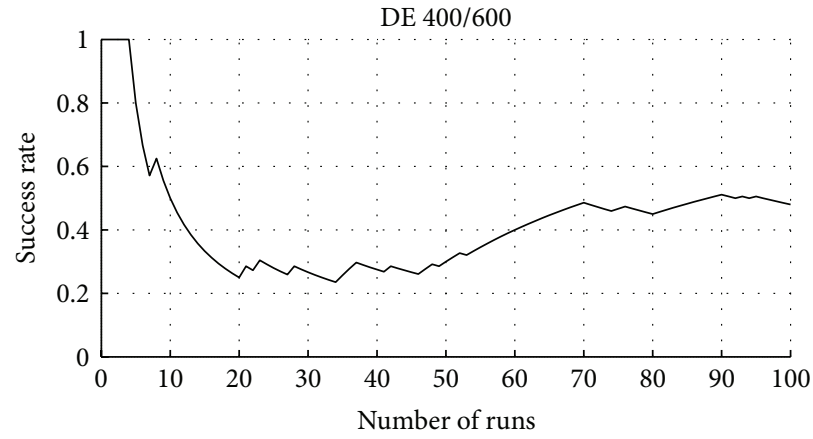

(a) Success rate for JEO MGADSM case

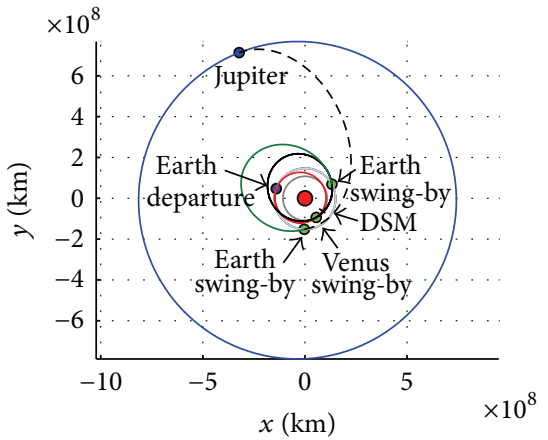

(b) JEO mission trajectory

FIGURE 6: The success rate and the trajectory for the JEO MGADSM case.

(EVVEJS)) [13]. The SSDM for this solution is 5. A local optimizer is used to tune the design variables to the nearest local minimum. The obtained mission total cost is $12.32 \mathrm{~km} / \mathrm{s}$. Table 10 shows the obtained solution design variables values. Figure 7 shows the zero-DSM trajectory.

Statistics are generated on the behavior of the HGDE over 200 runs and for 100,000 function evaluations. The success rate in finding the swing-by sequence is $58 \%$, whereas the success rate in finding the minimum mission cost is $55 \%$, as shown in Figure 8(a).

The second step (the MGADSM model) is carried out assuming the EVJS sequence. The boundaries of the design variables in the second step are shown in Table 11. Several sizes for the population are tried; these are $\mathrm{NP}=$ $60,90,150,400$, and 600 . In all of these cases, the HGDE could not find a lower cost solution than the zero-DSM model. Hence, the best solution obtained by the HGDE for the sequence EVJS is $12.32 \mathrm{~km} / \mathrm{s}$.

In [13], a niching approach was implemented in the HGGA to maintain population diversity in subsequent generations, by degrading the fitness of the similar individuals [26]. As a result, the HGGA was able to find the known optimal sequence (EVVEJS) for the Cassini 2 mission. The same niching approach is implemented in HGDE for the

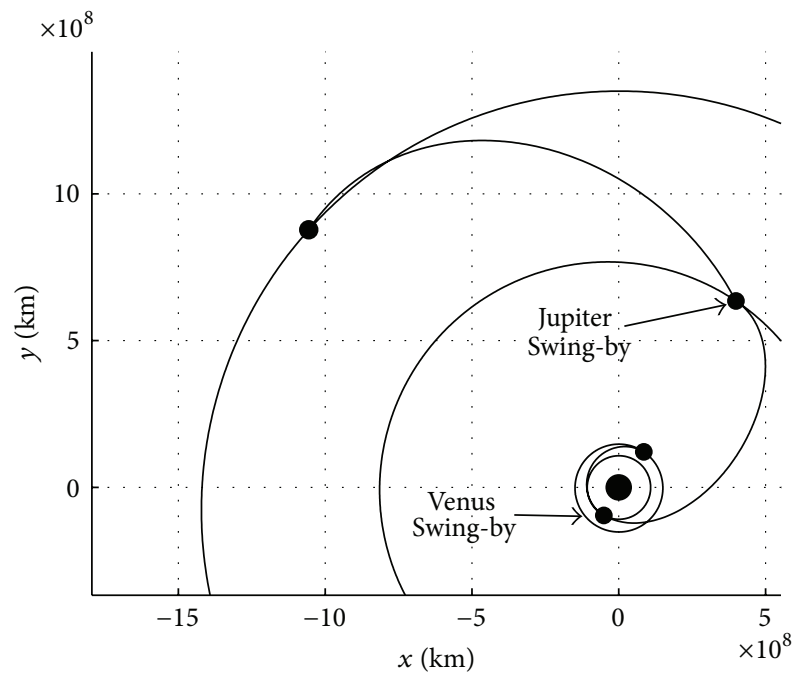

FIgURE 7: Optimal Cassini 2 mission: zero-DSM model.

Cassini 2 mission. However, the HGDE still could not find a sequence better than the EVIS. The resulting success rates, when niching is implemented, are shown in Figure 8(b). 


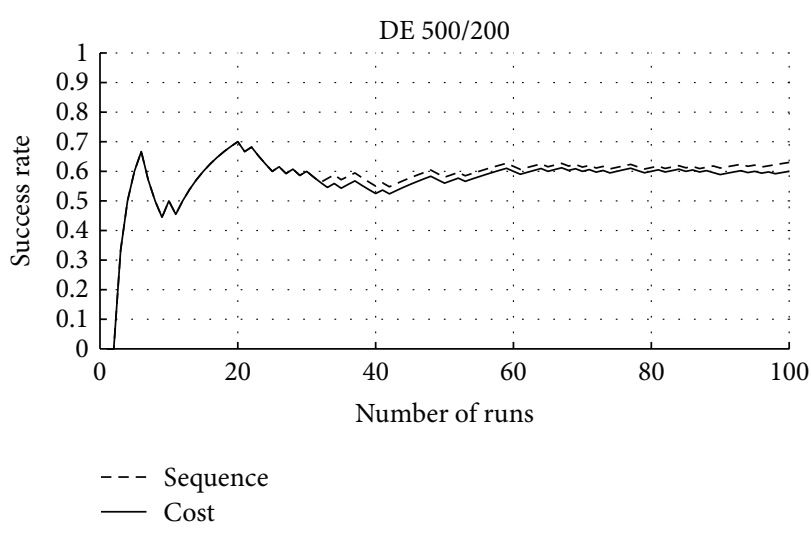

(a) Zero-DSM case

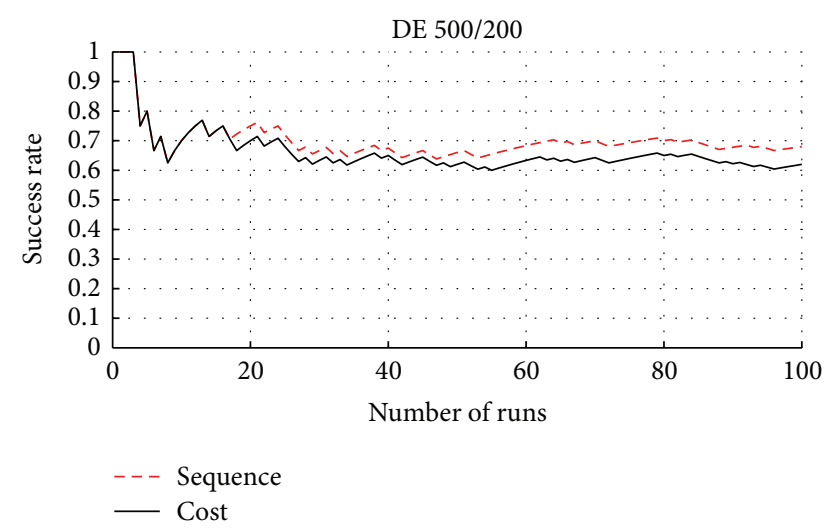

(b) Zero-DSM case with niching

FIGURE 8: The success rate for finding the optimal cost and swing-by sequence, for the Cassini 2 mission.

TABLE 10: Zero-DSM model trajectory of Cassini 2 mission using HGDE.

\begin{tabular}{lc}
\hline Mission parameter & Zero-DSM Model \\
\hline Departure date & 16-Nov-1997 13:55:12 \\
Departure impulse $(\mathrm{km} / \mathrm{s})$ & 4.5594 \\
Venus swing-by date & 03-Apr-1998 21:55:12 \\
Pericenter altitude $(\mathrm{km})$ & 605.19 \\
Jupiter swing-by date & 25-Aug-2000 21:55:12 \\
Postswing-by impulse $(\mathrm{km} / \mathrm{s})$ & 0 \\
Pericenter altitude $(\mathrm{km})$ & 1285964 \\
Arrival date & 01-Jan-2007 23:45:36 \\
Arrival impulse $(\mathrm{km} / \mathrm{s})$ & 3.4 \\
Time of flight $($ days $)$ & 138.3, $875,2320.1$ \\
Mission duration $(\mathrm{days})$ & 3333.4 \\
\hline Mission cost $(\mathrm{km} / \mathrm{s})$ & $\mathbf{1 2 . 3 1 5}$ \\
\hline
\end{tabular}

Another experiment is conducted to solve the zero-DSM model for the Cassini 2 mission. In this experiment, the number of swing-bys is assumed to range from 3 to 5 . This range is selected to exclude the previously obtained two swing-by solutionS from the design space. This experiment is conducted 100 times. The obtained zero-DSM solution has three swing-bys (planet sequence is EVEJS.) The SSDM for this solution is 1 . This zero-DSM solution has a cost of $10.15 \mathrm{~km} / \mathrm{s}$. The success rates in finding this solution ARE $25 \%$ and $27 \%$ for the cost and swing-by, respectively.

Assuming that the planet sequence is known (EVVEJS), the HGDE is used to solve the optimal DSM structure in the trajectory. The number of design variables in this case is 48 (6 discrete and 42 continuous). The range for the time of flight of each leg is assumed to be 40 to 1000 days. The DE 500/200 experiment is conducted 100 times. The obtained solution has a single DSM in the second leg (VV), whose impulse is $0.469 \mathrm{~km} / \mathrm{s}$. The total mission cost is $8.89 \mathrm{~km} / \mathrm{s}$. The success rate in finding this solution is $6 \%$. The obtained solution is shown in Table 12.

\section{Discussion}

The main advantages of implementing the HGDE in this problem are (1) its capability in finding the optimal swingby sequence and the optimal number of DSMs, and (2) the zero-DSM solution provides good initial guesses for some of the design variables which increases the effectiveness of the second step of HGDE in finding the minimum cost MGADSM trajectory.

The Earth-Mars mission is solved using the HGDE in this paper and is solved using the HGGA in [12]. The best solution found using the HGGA has a cost of $10.78 \mathrm{~km} / \mathrm{s}$, while the best solution found using the HGDE has a cost of $10.73 \mathrm{~km} / \mathrm{s}$. The success rate of the HGDE in finding the optimal swing-by sequence is $30 \%$ and is $20 \%$ in finding the minimum cost, when the MGADSM problem is solved in one step. The same experiment was conducted using the HGGA, and the success rate was $1.5 \%$ in finding the swingby sequence and was $10 \%$ in finding the minimum cost [12]. The same problem was solved on two steps, the zeroDSM and MGADSM, using both the HGGA and the HGDE. The success rates of the HGGA are 95\% and 25\% for the swing-by and cost, respectively. The corresponding success rates for the HGDE are $100 \%$ and $90 \%$ for the swing-by and cost, respectively. Clearly, the HGDE has higher success rates. Moreover, the efficiency of the HGDE is much higher than that of the HGGA. The HGDE needed 6,000 functions evaluations, while the HGGA required 150,000 functions evaluations, to obtain the above success rates. Without using the concept of hidden genes, the problem can only be solved assuming the knowledge of the swing-by sequence and the number of DSMs in the solution. For instance, assuming a swing-by planet Venus and one DSM, [2] found a solution with a cost of $10.786 \mathrm{~km} / \mathrm{s}$.

The Jupiter Europa Orbiter Mission trajectory from Earth to Jupiter has been optimized in the literature. The solution presented in this paper is the same as the solution obtained by the HGGA [14]. Reference [14] presents another slightly lower cost solution that is obtained using a dynamic-size multiple populations genetic algorithms [14]. This slightly lower cost 
TABLE 11: Bounds of Cassini 2 mission's design variables: second optimization phase.

\begin{tabular}{lcc}
\hline Design variables & Lower bound & Upper bound \\
\hline No. of DSMs in each mission's leg, $n_{1}, \ldots, n_{i+1}$ & 0 & 2 \\
Flight direction, $f$ & Prograde & Retrograde \\
Departure date, $t_{d}$ & 10 -Nov-1997 & 20 -Nov-1997 \\
Arrival date, $t_{a}$ & 01 -Dec-2006 & $30-$ Mar-2007 \\
Time of flight (days)/leg, $T_{1}, \ldots, T_{i}$ & $125,800,2000$ & $170,950,2500$ \\
Swing-by normalized pericenter altitude, $\bar{h}_{1}, \ldots, \bar{h}_{i}$ & 0.1 & 10 \\
Swing-by plane rotation angle $(\mathrm{rad}), \eta_{1}, \ldots, \eta_{i}$ & 0 & $2 \pi$ \\
Epoch of DSM, $\varepsilon_{1}, \ldots, \varepsilon_{j}$ & 0.1 & 0.9 \\
DSM $(\mathrm{km} / \mathrm{s}), \Delta v_{1}, \ldots, \Delta v_{k}$ & -5 & 5 \\
\hline
\end{tabular}

TABLE 12: MGADSM trajectory of Cassini 2 mission using HGDE.

\begin{tabular}{lc}
\hline Mission parameter & MGADSM model \\
\hline Departure date & 03-Nov-1997 15:39:21 \\
Departure impulse $(\mathrm{km} / \mathrm{s})$ & 3.9141 \\
Venus swing-by date & 30-Mar-1998 06:26:22 \\
Pericenter altitude $(\mathrm{km})$ & 674.8 \\
DSM date & 17-Nov-1998 08:04:14 \\
DSM impulse $(\mathrm{km} / \mathrm{s})$ & 0.46911 \\
Venus swing-by date & 13-Jul-1999 04:43:10 \\
Pericenter altitude $(\mathrm{km})$ & 605.2 \\
Earth swing-by date & 23-Aug-1999 20:53:59 \\
Pericenter altitude $(\mathrm{km})$ & 3588.16 \\
Jupiter swing-by date & 06-Apr-2001 01:02:28 \\
Pericenter altitude $(\mathrm{km})$ & 4605630.3 \\
Arrival date & 21-May-2007 16:47:46 \\
Arrival impulse $(\mathrm{km} / \mathrm{s})$ & 4.2449 \\
Time of flight $($ days $)$ & 146.6, 469.9, 41.6, 591.1, 2236.6 \\
Mission duration $(\mathrm{days})$ & $\mathbf{8 . 8 9}$ \\
\hline Mission cost $(\mathrm{km} / \mathrm{s})$ &
\end{tabular}

solution has a cost of $8.92 \mathrm{~km} / \mathrm{s}$ and has a different planet sequence of only two swing-bys (EEEJ) and three DSMs [14].

The Cassini 2 mission trajectory design problem has been addressed in several studies $[2-4,6]$, where it is always assumed that a fixed swing-by sequence (EVVEJS) is known a priori. Different methods have been used in the literature that result in slightly different costs for the Cassini 2 mission; one solution reported in [2] has a cost of $8.877 \mathrm{~km} / \mathrm{s}$. The solution obtained by the HGGA has a cost of $8.385 \mathrm{~km} / \mathrm{s}$, when the problem is solved in two steps. The HGGA solution is only possible when a niching approach is implemented [13]. The HGDE is implemented for solving the Cassini 2 mission. Even with niching, the HGDE could not find the known planet sequence. This is because the implemented niching approach is originally designed to maintain diversity in GA populations and might not be the best approach to maintain diversity in DE populations.

The results presented in this paper show that the HGDE algorithm is more efficient in searching for the optimal solution of the MGADSM problem than the HGGA. This is consistent with previous results in the literature where the
DE method has proven to be more efficient than the GA in solving the same problem when the number of design variables is fixed [15].

\section{Conclusion}

The concept of hidden genes is implemented in differential evolution for solving the interplanetary trajectory optimization problem. In the interplanetary trajectory optimization problems presented, the HGDE automatically computes, without a priori knowledge, the number of swing-bys, the planets of swing-bys, and the number of deep space maneuvers, in addition to finding the rest of the trajectory parameters. The HGDE implementation is simpler than other evolutionary algorithms that carry out genes exchange between different length chromosomes. This is because the HGDE implements the standard crossover operation. In all case studies presented in this paper, the HGDE has demonstrated to have an advantage, as compared to the HGGA, in terms of the success rate of the algorithm. However, in the Cassini 2 mission trajectory, a niching approach added to the HGGA enabled finding the optimal solution while the HGDE, with niching, could not find the known optimal solution.

\section{References}

[1] D. Izzo, V. M. Becerra, D. R. Myatt, S. J. Nasuto, and J. M. Bishop, "Search space pruning and global optimisation of multiple gravity assist spacecraft trajectories," Journal of Global Optimization, vol. 38, no. 2, pp. 283-296, 2007.

[2] J. T. Olympio and J. P. Marmorat, "Global trajectory optimisation: can we prune the solution space when considering deep space maneuvers?" Final Report, European Space Agency, 2007.

[3] J. T. Olympio, "Designing optimal multi-gravity-assist trajectories with free number of impulses," in Proceedings of the 21st International Symposium on Space Flight Dyncamics, Toulouse, France, 2009.

[4] M. Vasile and P. de Pascale, "Preliminary design of multiple gravity-assist trajectories," Journal of Spacecraft and Rockets, vol. 43, no. 4, pp. 794-805, 2006.

[5] M. Ceriotti and M. Vasile, "Automated multigravity assist trajectory planning with a modified ant colony algorithm," Journal of Aerospace Computing, Information and Communication, vol. 7, no. 9, pp. 261-293, 2010. 
[6] A. D. Olds, C. A. Kluever, and M. L. Cupples, "Interplanetary mission design using differential evolution," Journal of Spacecraft and Rockets, vol. 44, no. 5, pp. 1060-1070, 2007.

[7] Y. H. Kim and D. B. Spencer, "Optimal spacecraft rendezvous using genetic algorithms," Journal of Spacecraft and Rockets, vol. 39, no. 6, pp. 859-865, 2002.

[8] H. Kim, O. Jung, and H. Bang, "A computational approach to reduce the revisit time using a genetic algorithm," in Proceedings of the International Conference on Control, Automation and Systems (ICCAS '07), pp. 184-189, Seoul, Republic of Korea, October 2007.

[9] O. Abdelkhalik and D. Mortari, "Orbit design for ground surveillance using genetic algorithms," Journal of Guidance, Control, and Dynamics, vol. 29, no. 5, pp. 1231-1235, 2006.

[10] T. A. Ely, W. A. Crossley, and E. A. Williams, "Satellite constellation design for zonal coverage using genetic algorithms," Journal of the Astronautical Sciences, vol. 47, no. 3-4, pp. 207-228, 1999.

[11] G. A. Rauwolf and V. L. Coverstone-Carroll, "Near-optimal lowthrust orbit transfers generated by a genetic algorithm," Journal of Spacecraft and Rockets, vol. 33, no. 6, pp. 859-862, 1996.

[12] O. Abdelkhalik, "Hidden genes genetic optimization for variable-size design space problems," Journal of Optimization Theory and Applications, vol. 156, no. 2, pp. 450-468, 2013.

[13] A. Gad and O. Abdelkhalik, "Hidden genes genetic algorithm for multi-gravity-assist trajectories optimization," AIAA Journal of Spacecraft and Rockets, vol. 48, no. 4, pp. 629-641, 2011.

[14] O. Abdelkhalik, "Multi-gravity-assist trajectories optimization: comparison between the hidden genes and the dynamic-size multiple populations genetic algorithms," in Proceedings of the AAS/AIAA Astrodynamics Specialist Conference, No. AAS11-620, Girdwood, Alaska, USA, July-August 2011.

[15] M. Vasile, E. Minisci, and M. Locatelli, "Analysis of some global optimization algorithms for space trajectory design," Journal of Spacecraft and Rockets, vol. 47, no. 2, pp. 334-344, 2010.

[16] D. Izzo, Spacecraft Trajectory Optimization, vol. 29, chapter 7, Cambridge University Press, New York, NY, USA, 1st edition, 2010.

[17] M. Vasile and M. Ceriotti, Spacecraft Trajectory Optimization, vol. 29, chapter 8, Cambridge University Press, New York, NY, USA, 1st edition, 2010.

[18] O. Abdelkhalik and A. Gad, "Dynamic-size multiple populations genetic algorithm for multigravity-assist trajectories optimization," Journal of Guidance, Control, and Dynamics, vol. 35, no. 2, pp. 520-529, 2012.

[19] J. Zhang and A. C. Sanderson, Adaptive Differential Evolution, a Robust Approach to Multimodal Problem Optimization, vol. 1 of Adaptation, Learning, and Optimization, Springer, Berlin, Germany, 2009.

[20] K. Price, R. M. Storn, and J. A. Lampinen, Differential Evolution: A Practical Approach to Global Optimization, Natural Computing Series, Springer, Berlin, Germany, 2005.

[21] B. Starr, "Spooled DNA and hidden genes: the latest finding in how our DNA is organized and read," The Tech Museum of Innovation, Department of Genetics, Stanford School of MedicineSan Jose, Calif, USA, http://www.thetech.org/genetics/news.php?id=31.

[22] M. Vasile, E. Minisci, and M. Locatelli, "An inflationary differential evolution algorithm for space trajectory optimization," IEEE Transactions on Evolutionary Computation, vol. 15, no. 2, pp. 267-281, 2011.
[23] K. Clark, J. Boldt, R. Greeley et al., "Return to Europa: overview of the jupiter Europa orbiter mission," Advances in Space Research, vol. 48, no. 4, pp. 629-650, 2011.

[24] T. Sweetser, T. R. Maddock, J. Johannesen et al., "Trajectory design for a Europa orbiter mission: a plethora of astrodynamic challenges," in Proceedings of the AAS/AIAA Space Flight Mechanics Meeting, Plethora of Astrodynamic Challenges, Paper No. AAS 97-174, pp. 97-174, 1997.

[25] European Space Agency, "GTOP act trajectory database," 2009, http://www.esa.int/gsp/ACT/inf/projects/gtop/gtop.html.

[26] D. Beasley, D. R. Bull, and R. R. Martin, "A sequential niche technique for multimodal function optimization," Evolutionary Computation, vol. 1, no. 2, pp. 101-125, 1993. 

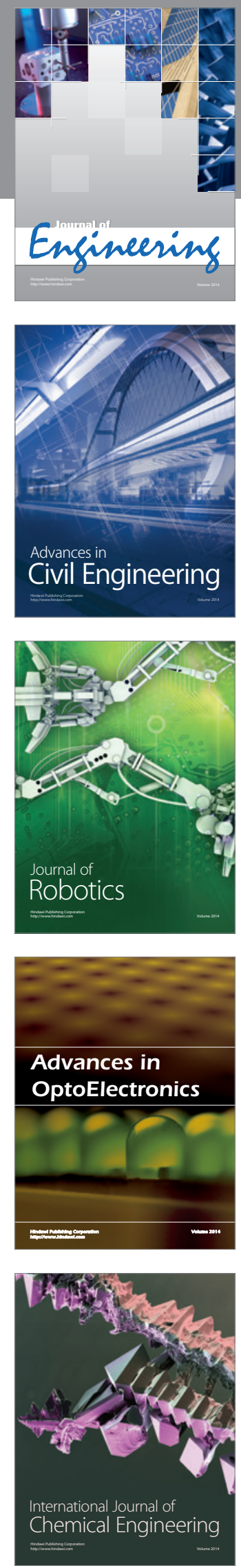

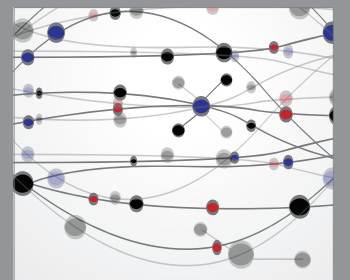

The Scientific World Journal
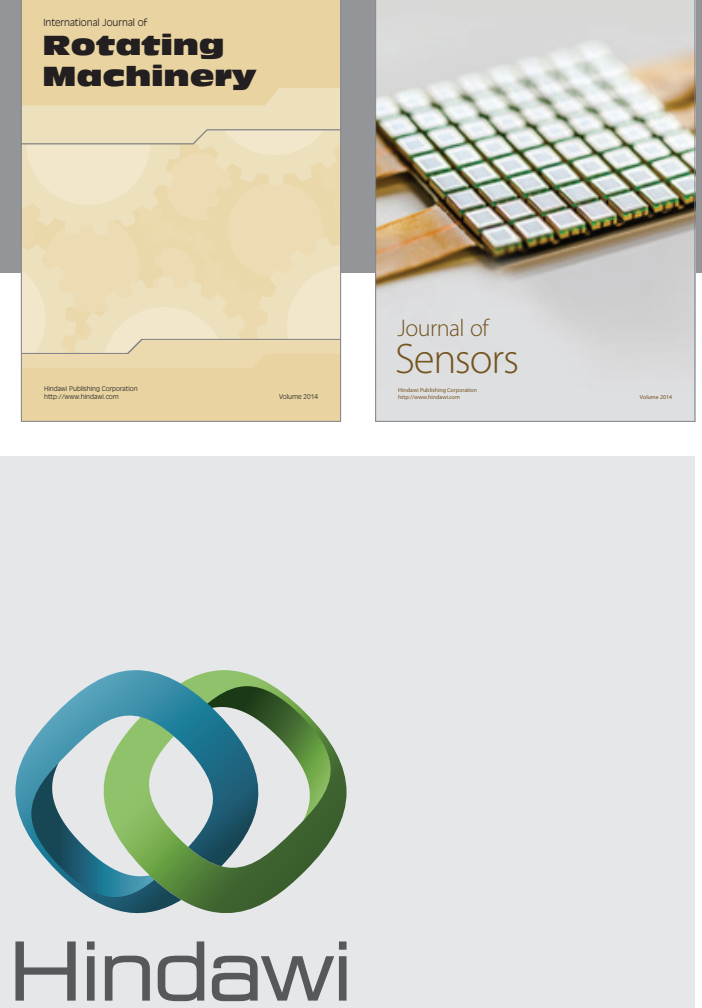

Submit your manuscripts at http://www.hindawi.com
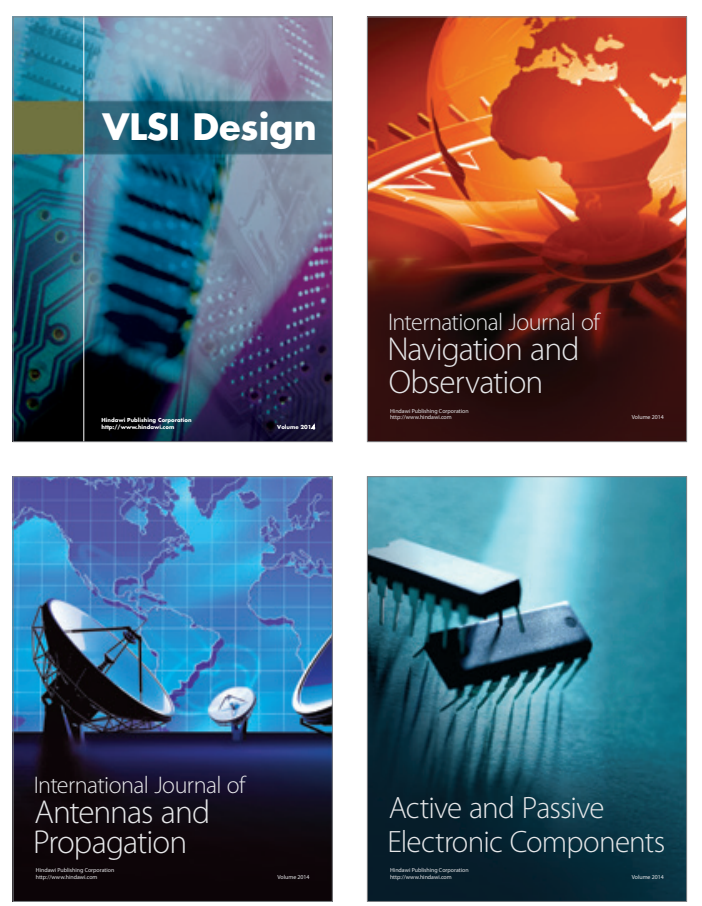
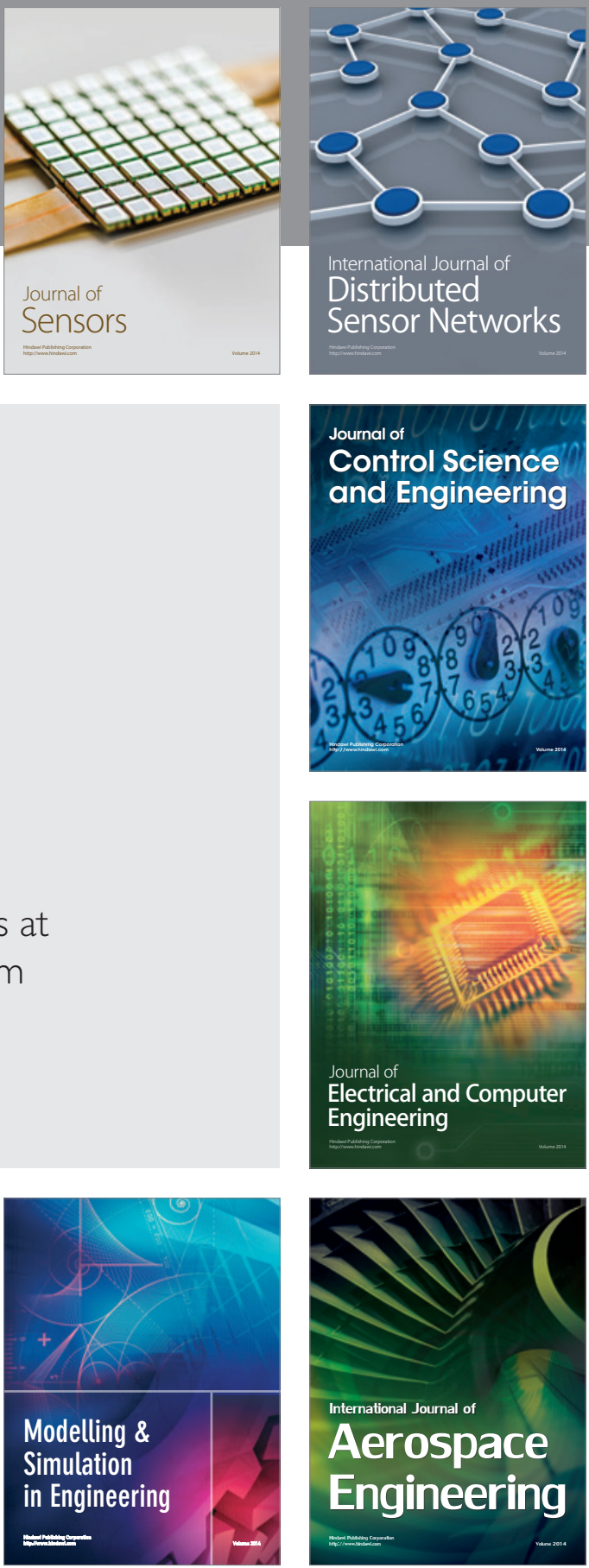

Journal of

Control Science

and Engineering
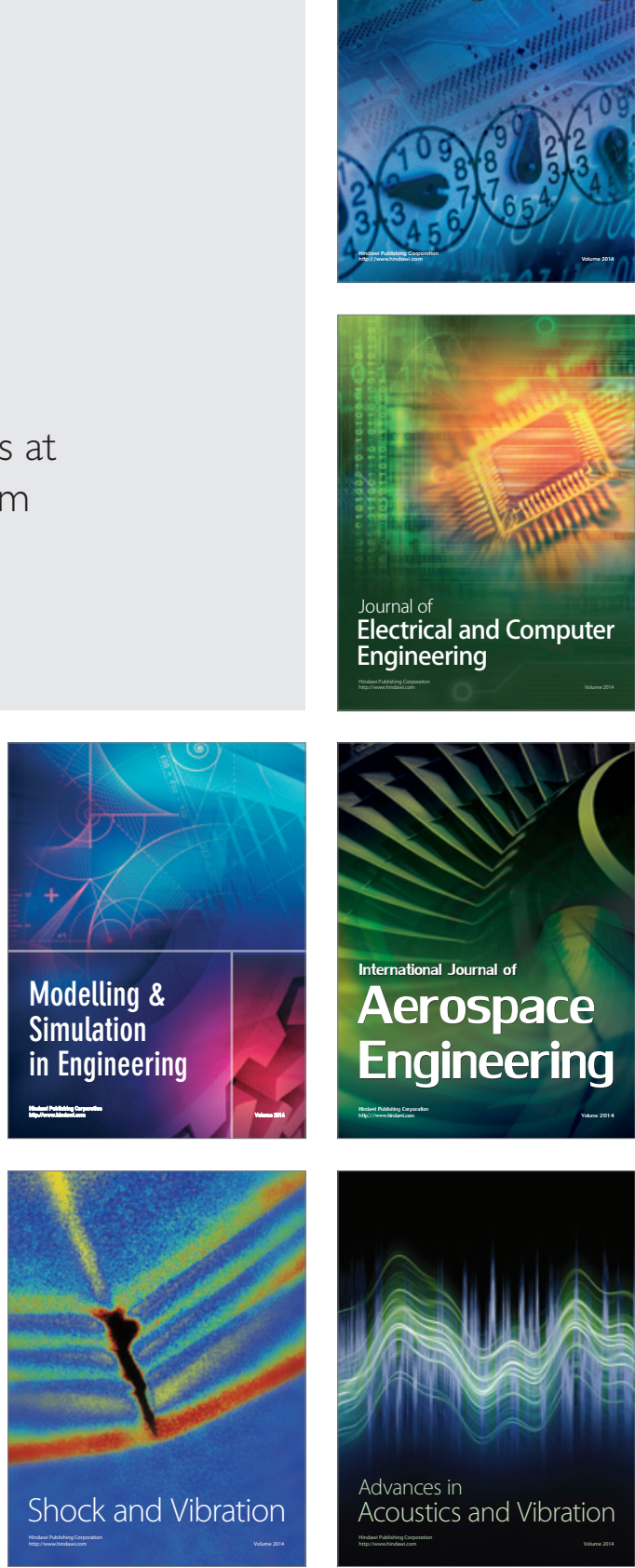